\title{
Potential environmental impacts associated with large-scale herbicide-tolerant GM oilseed rape crops
}

Oléagineux, Corps Gras, Lipides. Volume 11, Numéro 4, 246-52, JUILLET-OCTOBRE 2004, OGM

\author{
Auteur(s) : Antoine MESSÉAN, Marc FELLOUS \\ President of the CGB University of Paris 7 Denis Diderot Antoine Messéan Inra
}

Summary : The Biomolecular Engineering Commission considers that the knowledge acquired in the last three years has provided significant information in reply to the points raised in its review dated 16 February 2001.The Commission has studied the potential environmental impacts associated with large-scale herbicide-tolerant GM oilseed rape crops, making a distinction between direct and indirect impacts. Direct impacts stem from the intrinsic properties of herbicide-tolerant GM oilseed rape crops whereas indirect impacts result from practices associated with the farming of these crops.The Commission considers that, in the absence of the use of the herbicide in question in and outside of farmed land, there is no direct environmental risk (development of invasive crops per se) associated with the presence of a herbicide-tolerance gene in oilseed rape (or related species).Nevertheless, since the interest of these tolerant crops lies in the use of the herbicide in question, indirect effects, to varying extents, have been identified and must be taken into account: the use of the herbicide in question, applied to agricultural fields containing the herbicide-tolerant crop could lead to an increase in oilseed rape volunteer populations in crop rotations ; - the selective pressure exerted by non-specific herbicides (to which the crops have been rendered tolerant) may be very high in cases of continuous and uncontrolled use of these herbicides, and may result in the persistence of rare events such as the reproduction of fertile interspecies hybrids ; - the change to the range of herbicides used should be conveyed by more effective weed control and, like any change in farming practices, induce indirect effects on the agri-ecosystem, particularly in terms of changes to weeds and the associated animal life. Accordingly, the Biomolecular Engineering Commission recommends a global approach in terms of the large-scale farming of herbicide-tolerant crops that: accounts for the characteristics of the herbicide and its current and future use ; - accounts for herbicide-tolerant varieties belonging to other species, liable to be farmed in French agriculture in the short term ;- targets, if applicable, the selection of crop/herbicide combinations according to the constraints associated with French agriculture ; - coordinates the evaluations conducted on herbicide-tolerant crops and those conducted on herbicides ; - implements a biovigilance system and its associated resources whenever herbicide-tolerant crop farming is envisaged. Our report highlights the need for the management of any herbicide-tolerant GM oilseed rape crops. Such a management plan must account for the diverse cropping situations, including crop rotations and farming practices. Management should be based on the prior evaluation of situations encountered, the development of measures commensurate with the risks, and a validation of their efficacy through biovigilance.The Biomolecular Engineering Commission considers that the indirect environmental and agronomic impacts associated with current management practices employed for herbicide-tolerant oilseed rape crops can only be determined, in addition to the knowledge acquired, by continuing ongoing experimental studies initiated. Mathematical and computer models - by formalizing complex 
scenarios that incorporate the functioning of different oilseed rape populations (farmed, spontaneous, volunteer plants) under specific farming practices - enables the simulation of potential impacts and the identification of suitable management measures. Nevertheless, at the present time, the introduction of more extensive farming than that currently practised, or managed progressive introduction, would make it possible to progress in the study of impacts and develop and validate management procedures enabling the limitation of adverse impacts.In the specific case of imports, the Biomolecular Engineering Commission considers that herbicide-tolerant GM oilseed rape seed imports for processing and animal feed does not represent additional risks for the environment compared to other oilseed rape varieties on the market. Due to the very low likelihood of the dispersal of tolerant oilseed rape crops from imported seed, the impact of the herbicides used on communication routes is considered to be negligible in the current context. However, in order to supplement the information available on environmental impacts and avert any changes to the current situation, the Commission recommends that a general monitoring plan be implemented in parallel to the introduction of seeds onto the market.Finally, the Biomolecular Engineering Commission considers that biovigilance systems adapted to the various risks should be in place before any farming of commercial herbicide-tolerant varieties is undertaken.The Biomolecular Engineering Commission considers its analysis to be valid for non-selective herbicide-tolerant varieties of oilseed rape obtained both by transgenesis and by means of a technique other than transgenesis.

Keywords : Biomolecular Engineering Commission, environmental risks

\section{ARTICLE}

\section{Background and subject of order}

Within the scope of an official order from the French Ministry of the Environment and Agriculture, dated 6 July 2000, the Biomolecular Engineering Commission (CGB) conducted an evaluation of the environmental risks associated with the possible large-scale employment of herbicide-tolerant GM oilseed rape crops ${ }^{\underline{1}}$.

This evaluation resulted in a comprehensive report, dated 16 February 2001, in which the Commission concluded that there was no direct environmental risk (development of invasive crops per se) associated with the presence of a herbicide-tolerance gene in oilseed rape (or in related Brassica species), but stressed the need for additional information on the following points:

- - the effects generated by larger pollen source areas than those used to obtain the information available on oilseed rape pollen dispersal;

- - long-distance pollen dispersal and the role played by insects in particular;

- - better characterisation of interspecies hybrids (particularly amphidiploids) to aid in our understanding of species dynamics; 
- - the impact of persistent oilseed rape blocks, including those resulting from seed escaping during transport stages on the pollination of neighbouring oilseed rape and the dynamics of these blocks (persistence, extension, etc.).

The Biomolecular Engineering Commission also considered that large-scale experiments should be conducted in order to validate the management measures envisaged, intended to limit the direct and indirect effects of oilseed rape volunteer plants expressing the in herbicide-tolerance event.

Finally, the Biomolecular Engineering Commission recommended the initiation of a general study on the detailed analysis of the environmental impact of new weed control practices, based on the use of full-spectrum herbicides.

This evaluation provided a scientific basis for the implementation of a safeguard clause (article 16 of directive 90/220), making it possible to continue the provisional national suspension, in 2001, of the marketing of herbicide-tolerant GM oilseed rape, authorised at the European community level.

Within the scope of an order dated 26 June 2003, the French Ministry of Agriculture (the General Alimentation Division) requested that the Biomolecular Engineering Commission identify any possible new information liable to supplement or to call into question the scientific information previously used as a basis for the safeguard clause.

In an initial review dated july 2003, the Biomolecular Engineering Commission noted that, despite difficulties in completing the studies recommended in 2001 and the fact that some of these studies had not been finalised, significant new information was available and the Commission expressed the necessity to conduct a detailed analysis of this information before issuing a formal review.

\section{Restrictions of evaluation conducted}

In its analysis, the Biomolecular Engineering Commission restricted itself to herbicide-tolerant GM oilseed rape varieties. The analytical information reported below should be supplemented, or amended, for transgenic oilseed rape varieties with characteristics other than herbicide-tolerance (disease resistance, abiotic stress adaptation, oil quality). Nevertheless, the Biomolecular Engineering Commission considers its analysis to be valid for non-selective herbicide-tolerant varieties of oilseed rape obtained by means of techniques other than transgenesis.

Importantly, this analysis accounts for current farming practices in which crop weed control (using various techniques) plays an important role. The environmental effects of chemical weed control on plots are only discussed here in view of the fact that the use of GM crops results in changes to these practices.

A scientific research seminar, held on 28 November 2003, made it possible to summarise this information ${ }^{2}$ on each of the points mentioned in the review issued in February 2001 in view of the results obtained over the last three years and to initiate a more general study on indirect impacts and developments in weed control strategies associated with the use of non-selective herbicides.

This review reiterates the main queries raised in 2001 and provides answers obtained by the Commission from the scientific knowledge obtained since then. In addition, this review examines in more detail the issue of indirect impacts associated with the large-scale farming of herbicide-tolerant 
GM oilseed rape varieties, particularly on the basis of the lessons to be learned from the North American experience and the UK study on the impact on biodiversity. Finally, the specific case of the potential environmental impacts of herbicide-tolerant $\mathrm{GM}$ oilseed rape seed imports is discussed.

\section{Problem status and concept definition}

\section{Oilseed rape weed control, contribution of non-selective herbicide-tolerant crops}

Weed control is a widespread practice on agricultural land. Its purpose is to improve crop quality and crop yields by limiting the presence of competing plant species.

Phytopharmaceutical products currently play a predominant role among weed control techniques. The molecules employed are either selective and active against a given set of plants (either monocotyledons or dicotyledons) or non-selective and active against a range of plants. Depending on the molecules in question, the spectrum of action is more or less broad and the efficacy of the molecules is higher or lower.

In the case of oilseed rape, weed control treatments are commonplace. Weed control for oilseed rape is complex due to the presence of other, undesirable Brassica genus species with similar growth cycles to oilseed rape. Weed control strategies aiming to remove weeds from the crops after the oilseed rape crops have started to grow are currently very limited although they would enable the more rational use of herbicides. A range of diversified products exists, but their efficacy is targeted at Graminaceae and only some dicotyledons.

The introduction of tolerance to a non-selective herbicide makes it possible to use herbicidal products that can be applied after self-propagating plants ("weeds") have been removed effectively and should enable farmers to achieve more rational chemical weed control. This weed control strategy combined with herbicide-tolerant oilseed rape varieties would make it possible to reduce the number of treatments and the quantities of active substance sprayed per unit of surface area (estimated to be $30 \%$ - source CETIOM). According to CETIOM, the use of herbicide-tolerant oilseed rape could solve the serious weed control problems faced by farmers on $20 \%$ to $40 \%$ of agricultural land used for oilseed rape.

Non-chemical weed control practices also exist, generally employed as part of integrated crop weed protection. These include mechanical weed control and tillage, crop rotation, and sowing date. Integrated weed control involves moving from pure chemical curative weed control in a single farming year to overall control in terms of the succession of crops and integrating several weed control methods ${ }^{3}$. Integrated weed control consists of implementing all the technical, biological and biophysical factors contributing to limiting damage to the focal crop and subsequent crops in the rotation, in order to limit the use of curative herbicidal treatments and to reduce the environmental impacts associated with chemical residues.

Research on integrated weed control demonstrates not only the genuine efficacy of non-chemical practices in terms of the farming year, but also their high cost and their lower efficacy, both in the long-term and on other weed species. Rational chemical weed control appears to be an effective solution to supplement non-chemical practices by making it possible to control some weeds which 
are difficult to control by other means and providing solutions in years which are not favourable for integrated practices for climatic reasons. Within this context, the introduction of herbicide-tolerant transgenic crops could play an important, but non-systematic, role.

\section{Classification of potential impacts of herbicide-tolerant varieties}

There are two types of potential impacts of herbicide-tolerant varieties: environmental and agronomic. In each, a distinction will be made between direct and indirect impacts.

In environmental terms, the potential proliferation of the tolerant crop in ecosystems due to new breeding properties or the capacity for environmental adaptation (acquisition of a selective advantage) is considered to entail a direct impact. The acquisition of tolerance by related wild species and the potential for proliferation that would result are classified in the same way.

On the other hand, impacts associated with changes in herbicide residue levels in extensive agricultural areas of one or more crops tolerant to the same herbicide are indirect when there are changes in the weed populations and associated animal life. In such cases there may be selection of resistant forms associated with repeated herbicide application (and not due to gene transfer).

In agronomic terms, the multiplication of volunteer plants or weeds tolerant to one or more herbicides on agricultural land could impair farmers' abilities to ensure weed control over intercropping periods in rotation crops or in areas where these herbicides are applied. The severity of the impacts will be related, in particular, to the cumulative effects stemming from the use of several crops tolerant to the same herbicide in rotation.

These agronomic impacts raise the question of the sustainability of this weed control strategy and the herbicides corresponding to the tolerance introduced into the crops. This question involves aspects of production management, organisation, and licensing.

\section{New information relating to gene flow in oilseed rape}

\section{Pollen dispersal}

Oilseed rape produces large numbers of small seeds (on the order of one thousand seeds per plant). This species has a combined reproduction system with partial allogamy using an anemophilous and entemophilous process. Among insects, bees are considered to the main carriers of oilseed rape pollen. The reproductive biology varies depending on the pedo-climatic conditions and varieties.

\section{Pollen dispersal}

In oilseed rape, pollen dispersal is a complex dynamic process. Oilseed rape is particularly allogamous and, on average, an oilseed rape plant can be fertilised at a rate on the order of $30 \%$ by pollen produced by other oilseed rape plants. In addition, about $50 \%$ of the pollen produced by the plant is dispersed within a $3 \mathrm{~m}$ radius.

Pollen dispersal dynamics have been determined in a number of studies conducted in France and internationally using systems to quantify the role of the pollen from a "source" plot in the fertilisation of plants located at a given distance from this source. These studies demonstrate a decrease in gene flow with distance. Studies conducted on several thousand square metres in a 
continuous environment have demonstrated that the rate of seeds produced by fertilisation involving pollen produced by a given source fell below $1 \%$ over a distance on the order of $30 \mathrm{~m}$ and was approximately $0.1 \%$ over one hundred metres. Beyond $100 \mathrm{~m}$, this rate decreases very gradually with distance. For plants located over one $\mathrm{km}$ from the source, fertilisation rates are below $0.1 \%$ but not equal to zero. Observations made in Australia demonstrated occasional pollination at distances of a few kilometres. Nothing is known about pollination over larger distances in Austrailia. However, in a UK study, the use of isolated sterile male plants to detect pollen showed pollen dispersal at a distance of over $20 \mathrm{~km}$ from the source plot.

Variability with respect to the mean values mentioned here is very high. The pollination rate of the plants from one plot by plants from surrounding plots depends on the relative quantities of pollen from the recipient plot and surrounding plots. Specifically, it depends on the ratio between the size of the source and the size of the recipient plot, flowering synchronisation and male fertility. The fertilisation rates given above relate to fertile male varieties, i.e., those commonly used in commercial production. However, it has been observed that cross-pollination rates are higher when the recipient plants used are sterile males (or composite sterile male-pollinating hybrid varieties with only $20 \%$ fertile plants), which is also explained by the flowering biology (the length of the flowering period increases when low quantities of pollen are received). In addition, the pollen disperal over long distances is also subject to variable factors including landscape heterogeneity (hedges, rivers, etc.), climatic conditions, etc.

\section{Role of pollinating insects}

The role of insects, particularly bees, in oilseed rape pollination has been the subject of several studies in recent years.

In a study of the behaviour of bees with respect to glufosinate-ammonium-tolerant oilseed rape and its non-transgenic equivalent, no difference was observed either in terms of behaviour or in terms of honey-gathering, with both types of pollen being transported.

Pollinating insects appear to play an important role in the dispersal of pollen and in fertilisation. On the other hand, their direct contribution to long-distance transport appears to be limited and determined by the pollen exchanges liable to occur in beehives.

In sum, the major points demonstrated on pollen dispersal are as follows:

- - pollen dispersal declines rapidly with distance and then appears to follow a more uniform pattern, i.e. dispersal occurs, but at a very low rate (a few per thousand);

- - the dispersal rate does not appear to decline markedly at long distances. At least based on observations, there is no distance beyond which no pollen dispersal is observed;

- - the quantity of pollen produced by a plot of land depends on a number of factors: biological, climatic and agronomic;

- - the relative quantities of pollen produced by plots are a decisive factor in the crosspollination rate between the plots in question; 
- - pollinating insects play a role in long-distance pollination by favouring effective pollination at the plant level; they only appear to play a role in the transport of pollen from one plot to another through pollen exchanges liable to occur in beehives;

- - this pollen dispersal can be modelled and different models are currently being compared with the aim of making realistic predictions.

\section{Dynamics and impact of volunteer plant populations}

The dispersal of oilseed rape seeds occurs before and after harvesting in the crop plot and while the seeds are being transported between the plot and storage silos.

\section{Volunteer plant dynamics in plots}

The functioning of these field volunteer plant populations is now relatively clear. A high volume of seeds (1000 to 6000 seeds per $\mathrm{m}^{2}$ i.e. approximately 100 times the sowing dose) remains in the soil of the crop plot after harvesting. Once deeply embedded in the soil, these seeds remain viable, possibly for several years (between 5 and 10 years on average) and can regrow in the rotation crops. Volunteer plants then represent a potential new source of pollen and seed emission. The number of volunteer plants per unit of surface area depends particularly on the moisture content of the soil and farming practices during the period between crops. The size and fertility of these volunteer plants decreases with the vigour of the farmed crops with which they are in competition. Their frequency in subsequent oilseed rape crops appears to decline with the length of the rotation; short rotations (less than or equal to 3 years) do not generally guarantee presence levels of less than $1 \%$.

The effect of tillage has been evaluated in several studies and tillage methods appear to favour the germination of the seeds remaining in the soil in the period between crops following the harvest. In this case, the persistence of the seeds is decreased significantly.

Volunteer plant control is a practice already known to farmers who implement specific management measures for effective control. Nevertheless, in the case of herbicide-tolerant varieties, the repeated use of the herbicide in the rotation applies a high selective pressure on the tolerant crops. This may result in an increase in the number of seeds carrying tolerance in the soil and, for this reason, significantly more difficult volunteer plant control. Reinforcing volunteer plant control in rotation (tillage, suitable weed control) and the adoption of practices preventing the application of selective pressure on these volunteer plants is necessary.

Volunteer plant control would particularly increase management difficulties for farmers if the change involved tolerance to a herbicide already widely used for other agricultural purposes, or a herbicide widely used on another major crop used in the same rotations. The problem would not be so crucial for a herbicide which is not widely used or the use of which is restricted to oilseed rape.

\section{Dispersal of seeds outside plots}

Significant progress has been made in recent years in the knowledge of the functioning of volunteer plant populations on field edges, roads or in non-farmed areas in general, referred to as feral populations. 
To understand feral dynamics, it is important to account for four different types of populations: volunteer plants in fields, farmed crops, feral populations and volunteer plants in fallow land. In fact, the flowering dates, and therefore potential pollen flow dates, between these four population types overlap.

Some feral populations have surprisingly long life cycles (at least eight years in the Selommes study in the Centre of France). A large number of populations are not only the result of seeds falling from oilseed rape crop fields the previous year. In order to identify the factors determining their life cycle, a functional model of these populations, incorporating all the information available to date, has been elaborated. The three most important factors are the harvesting method, the herbicidal treatment, and the density. The presence of herbicide tolerance in these feral populations could induce an increase in the life cycle of these populations in the event of repeated use of the non-selective herbicide.

The impact of feral populations on the dispersal of herbicide-tolerance genes in the environment may prove to be non-negligible in the case of practices favouring their maintenance and growth. In this context, the application of selective pressure through the use of non-selective herbicides in the maintenance of these non-farmed areas represents a key factor. As in the case of volunteer plants in plots, the different types and uses of these herbicides must be taken into account.

\section{Production and selective value of interspecies hybrids}

Oilseed rape is a natural amphidiploid interspecies hybrid $(2 n=38)$ between two diploid species, Brassica oleracea (a cabbage) and Brassica rapa ssp campestris (bird seed rape). It belongs to a complex of phytogenetically similar species, which explains why interspecies hybridisations are possible. The likelihood of these hybridisations depends on several factors, in particular, the joint presence and flowering of the two potential parent species of the hybrid in the area in question. As such, the absence of certain species in French agricultural areas confirms that the likelihood of certain types of hybrids appearing is practically non-existent.

Regarding the two species from which oilseed rape originates, Brassica oleracea and Brassica rapa ssp. Campestris, hybridisations with oilseed rape are possible. However, Brassica rapa is only occasionally encountered in oilseed rape crop areas in France and, in addition, is not considered to be a weed in this crop. For this reason, hybridisations observed in the UK and in some Northern European countries are highly unlikely in France. In the case of cabbage (Brassica oleracea) for which a high genetic variability exists, hybridisations are not always possible. In addition, as the farmed forms are kept at the vegetative stage during cultivation, the likelihood of hybridisation is also very low. Spontaneous forms of this species are known but their distribution in France is very limited.

Therefore, studies in France have focused on the analysis of the likelihood of obtaining three types of interspecies hybrids involving wild radish (Raphanus raphanistrum), hoary mustard (Hirschfeldia incana) or wild mustard (Sinapis arvensis). Oilseed rape rarely hybridises with wild radish, even more rarely with hoary mustard and not at all with wild mustard.

The fertilisation of wild radish flowers by oilseed rape pollen produces hybrid seeds at an estimated frequency between $10^{-7}$ and $310^{-5}$, i.e. between one seed per ten million and 3 seeds per 100,000 . In 
the opposite direction, wild radish pollen fertilises neighbouring oilseed rape flowers at a frequency between $210^{-5}$ and $510^{-4}$. The characteristics of the genomic structures of several generations of hybrids demonstrates that there is no introgression of oilseed rape genes in the wild radish genome: the complex genetic structures produced consist of variable proportions of unaltered (by recombination) chromosomes of both species.

Depending on whether the gametes are reduced or not, hybridisations may generate diploids, triploids or amphidiploids. The rare "amphidiploids" with 56 chromosomes detected under farming conditions with wild radish have been studied more specifically in terms of their genetics and biology over the past three years. It has emerged that, in fact, they do not comprise wild radish chromosomes, but they can be fertilised by wild radish, making it possible to generate lineages. The selective value of hybrids varies but is always found to be lower than that of oilseed rape.

Hybridisation between oilseed rape and wild radish is possible but the frequencies obtained so far have been very low. Due to their lower selective value, the impact of interspecies hybrids in ecosystems is negligible in the absence of selective pressures. Their actual impact will depend on farming practices and the use of non-selective herbicides.

In view of the low frequency of hybridisation events, it is somewhat illusive to attempt to determine occurrence and growth conditions with field experiments. Large-scale observations under normal conditions of use would make it possible to determine the frequency and conditions favouring the formation of such hybrids.

\section{Other impacts on agri-ecosystems associated with the farming of herbicide-tolerant GM oilseed rape}

\section{Impact on plant and animal life in agricultural ecosystems}

The indirect environmental impacts associated with possible changes to weed species and associated animals and with changes in herbicide residue levels in the environment need be taken into account for the potential use of one or more plant species tolerant to the same herbicides.

As illustrated by the recent "Farm Scale Evaluation" study in the UK intended to evaluate the impact of herbicide-tolerant crops on biodiversity, significant effects are expected in the trophic chain. On average, weed control for a glufosinate-ammonium-tolerant spring crop induces a significant reduction in the biomass of weeds throughout the crop cycle and a reduction in the seed stock time compared to weed control with conventional oilseed rape. This weed limitation has negative effects on associated organisms, particularly butterflies.

This result stems from the greater efficacy of the non-selective herbicide used and its conditions for use (such as the application date in the crop cycle) and is not a direct effect of the GM crop. Similarly, the introduction of herbicide-tolerant crops leads to changes in plant life and indirect effects on associated animal life, just as the use of effective conventional herbicides have induced changes in plant and animal life in the past. If the weed control practised on the herbicide-tolerant crop is more effective than weed control on the conventional crop, a reduction in weeds and their associated organisms will be observed on the plot itself and its immediate vicinity: this is the case for spring 
oilseed rape and beet in the UK study. All else being equal, the introduction of herbicide-tolerant oilseed rape crops would result in a marked decrease in weeds and the associated animal life in agricultural land allocated to oilseed rape.

It has also emerged from the UK study that the difference observed between the biodiversity encountered between crops is greater than that observed between GMOs/non-GMOs for the same crop. While a herbicide-tolerant spring oilseed rape crop associated with the use of the same herbicide for weed control results in a marked reduction in the plant and animal life present in the plot compared to a conventional oilseed rape crop, the plant and animal life present in spring oilseed rape plots observed in the UK remain more plentiful than in other crops, beet or maize.

Due to these indirect effects, the overall evaluation of environmental impacts must be conducted at regional scales, incorporating farmed plots and non-farmed areas, and integrated management may optimise the balance between agricultural production, environmental impacts and biodiversity.

\section{Impacts observed with commercial distribution in Canada}

The use of hHerbicide-tolerant GM oilseed rape has grown since 1996 to reach its current employment of about $75 \%$ of land used for oilseed rape crops. While the adoption criteria appear to be primarily linked with greater flexibility and improved harvest quality (fewer impurities), direct beneficial effects on the environment have been reported: reduction in soil herbicide levels and in tillage, which is favourable for the quantity of organic matter in soils and the reduction of energy costs associated with cropping operations.

It is important to note that land management in Canada is substantially different to that in Europe which limits possible analogies. In fact, Canada is made up of vast, fully preserved natural environments, as well as crop areas that are considered as industrial and in which the majority of weeds have been imported with the crops.

In terms of gene flow, the development of volunteer plants tolerant to one or more herbicides has been demonstrated. They have been controlled to date on agricultural land by applying inexpensive conventional selective herbicides.

In terms of indirect environmental impacts, the effect of the type of tillage on biodiversity appears to be more important than that of herbicides, which stresses the importance of overall crop system management.

\section{Evaluation of management measures for herbicide-tolerant GM oilseed rape crops}

The indirect environmental and agronomic impacts of large-scale herbicide-tolerant GM oilseed rape crops are linked with the use of these products and the change in associated farming practices. The dispersal of genes by pollen or seeds may lead, depending on situations and practices, to the development of tolerant plants which would call into question the benefits of the non-selective herbicides in question. Therefore, it appears to be necessary to implement management measures in proportion to the risks in order to keep the number of these undesirable plants as low as possible. 
These measures should meet two requirements:

- (1) develop or generalise practices reducing the persistence of undesirable plants in time and space;

- (2) prevent the application of a selective pressure on undesirable plants.

Several measures have been proposed:

- - after the oilseed rape harvest, allow the seed to germinate and wait for the first rains (and therefore the first growths) before tilling in order to favour further seed growth and thus reduce the seed stock; tillage must be restricted to the ujpper few centimetres of soil;

- - reinforce volunteer plant control practices in other crops in the rotation to prevent the seed stock from increasing;

- - remove other crops tolerant to the same herbicide from the rotation so as to make volunteer plant control easier; this will be easier if the "herbicide tolerance" strategy is only employed for some crops;

- - space crops at sufficient distances to limit pollination between plots;

- - review other uses of non-selective herbicides (drying, fallow land, upkeep of non-farmed areas) if the tolerance gene to these herbicides is introduced into crops.

These specific measures, along with others such as border maintenance or fallow land allocation, were evaluated by means of numerical simulation using a Genesys-Oilseed rape gene flow model. This model, developed by INRA in conjunction with CETIOM, makes it possible to estimate accidental presence levels in a harvest for a set of plots, a sequence of crops on each plot and the techniques associated with each crop in the rotation.

This model has been validated using gene flow data from field experiments. While some fine-tuning is required for the quantitative prediction, the model makes it possible to classify crop systems and crop techniques correctly according to their impact on the presence of GMO plants in agricultural landscapes.

The various simulations conducted demonstrate the extreme variability of the results according to the situations in question. The structure of the set of plots and the spatial allocation of crops have an essential impact which implies that a specific diagnosis should be conducted in each agricultural situation. In addition, these studies confirm the importance of selective pressure control (for other purposes, avoid applications of the herbicide used on its own) and the upkeep of non-farmed areas (fallow land, road borders).

As a more general rule, modelling makes it possible to define plot layout measures in time and space to limit cross-breeding between GM, conventional or organic crops. These models may also help implement biovigilance systems by identifying at-risk situations in which environmental impacts will be more likely and specifying the sampling measures. 


\section{Specific case of seed imports}

In the case of imports of herbicide-tolerant GM oilseed rape seeds for processing and use in animal feed, the environmental impacts were considered by the Biomolecular Engineering Commission as negligible in the current context. While the risk of accidental dispersal of oilseed rape seeds during the transport phase remains possible, the extent of these accidental events would be limited, in view of the restricted number of importation points of oilseed rape seeds from outside countries, and the fact that these seeds are generally triturated and processed at these points of entry. As a result, any oilseed rape seeds which end up in the soil during importation or transport cannot induce the growth of plants in sufficient numbers to result in invasion and damage to the environment or to conventional oilseed rape crops.

However, in order to supplement the information available for the prospective evaluation of the risks for public health and the environment, the Commission recommends that a general monitoring system be implemented in parallel to the introduction of the products on the market. This information should make it possible to supplement the knowledge available on environmental impacts and consolidate the arguments on the lack of long-term effects of these imports.

\section{Conclusions}

The various studies conducted over the past three years have supplemented the already considerable volume of information on the impact of herbicide-tolerant GM oilseed rape varieties on the environment.

With respect to the direct environmental risk, the presence of herbicide tolerance in oilseed rape does not give it a selective advantage in natural ecosystems where the herbicide is not used.

Similarly, the introduction of herbicide tolerance into species related to oilseed rape by hybridisation appears to be a rare and transitory event which does not give these species a selective advantage in natural ecosystems where the herbicide is not used.

As a result, the risk of the propagation of oilseed rape or hybrids tolerant to one or more herbicides in ecosystems will be linked with changes in the practices associated with herbicide-tolerant transgenic oilseed rape crops and the use of the associated herbicides. The same applies for the indirect environmental impacts on plant and animal life in agricultural areas, which will be linked to the change in the herbicides applied, their uses and the associated practices.

With respect to agronomic risk, the presence of herbicide tolerance in species related to oilseed rape does not appear to represent an insurmountable problem in weed control, provided that selective pressure is controlled with rational management practices.

However, herbicide-tolerant oilseed rape volunteer plants must be managed by specific measures in terms of the crop year and the cropping system. These measures need help limit their occurrence and also prevent selective pressures on these volunteer plants. Therefore, the Biomolecular Engineering Commission recommends that a management framework be defined before any largescale farming of commercial varieties of herbicide-tolerant oilseed rape. 
With respect to specific phenomena such as the future of interspecies hybrids, the dynamics of spontaneous plants or very long distance dispersal, it would appear that only the introduction of large-scale crops $^{4}$ (several dozen hectares in an agricultural region) or progressive introduction accompanied by suitable regulatory and scientific follow-up will make it possible for a further understanding in the future. Similarly, modelling, widely used over the last three years, must be compared to additional data in order to fine-tune the models developed.

Various management measures have been evaluated with simulations using models incorporating agricultural landscapes and practices. Due to the extreme variability of the situations, it does not appear to be possible to recommend a single and generalised framework of management measures but it is necessary to adapt these measures to each situation. Therefore, the Biomolecular Engineering Commission recommends the implementation of an approach based on prior analysis of the situations encountered, the definition of measures suited to these situations and validation of the efficacy of the measures through biovigilance.

With respect to the specific case of imports, the Biomolecular Engineering Commission considers that imports of herbicide-tolerant GM oilseed rape for processing and animal feed do not represent higher risks for the environment that those of other oilseed rape varieties found on the market. However, in order to supplement the information available on environmental impacts, the Commission recommends that a general monitoring system be implemented in parallel with the introduction of the products onto the market.

\section{General conclusion}

The Biomolecular Engineering Commission considers that herbicide-tolerant GM oilseed rape crops do not represent direct risks for the environment, but require specific management and monitoring measures so as to control the indirect environmental impacts and agronomic impacts associated with the presence of volunteer plants in crop systems.

The Biomolecular Engineering Commission recommends the implementation of an ongoing evaluation approach based on the limited introduction of herbicide-tolerant oilseed rape, accompanied by prior analysis of the situations encountered, the definition of measures suited to these situations, and the validation of the efficacy of measures through close biovigilance.

In addition, the Biomolecular Engineering Commission recommends an overall approach in terms of the large-scale farming of herbicide-tolerant crops:

- - accounting for the type of herbicide and its current and future use;

- - accounting for herbicide-tolerant varieties belonging to other species, liable to be farmed in French agriculture in the short term;

- - targeting, if applicable, the selection of crop/herbicide combinations according to the constraints associated with French agriculture;

- - coordinating the evaluations conducted on herbicide-tolerant crops and those conducted on herbicides; 
- - implementing a biovigilance system and its associated resources whenever herbicidetolerant crop farming is to be envisaged.

The Biomolecular Engineering Commission is prepared to assist with the completion of studies in this field, which should also involve the Toxic substance commission, the Phytopharmaceutical product approval committee, the Permanent technical selection committee and the Biovigilance committee.ARRAY(0x2e9d40)ARRAY(0x2e9d7c)4 In this case, it is important for the crops used to have characteristics of varieties suitable for introduction onto the market.1 This order also related to sugar beet.2 The proceedings of this seminar will be available shortly. 3 Such as tillage, the introduction of a spring crop into the rotation and the change of sowing dates in the case of foxtail. 